\section{Epigenetics: new possibilities for drug discovery}

\author{
"Although several epigenetic drugs have been approved by the \\ FDA and a number of clinical trials are in progress, there are still \\ numerous possibilities to develop novel drugs by targeting epigenetic \\ mechanisms."
}

First draft submitted: 20 January 2017; Accepted for publication: 27 January 2017; Published online: 31 March 2017

Keywords: DNA methylation $\bullet$ drug discovery $\bullet$ epigenetics $\bullet$ histone modifications $\bullet$ miRNA - RNA interference

Difficulties in discovery and development of novel drugs based on conventional methods have accelerated applications of alternative approaches. Automation and miniaturization of drug screening as well as utilization of structure-based drug development programs have provided certain new possibilities. However, although epigenetic mechanisms have already been described a long time ago, only fairly recently the extension of their involvement in disease and their potential in drug discovery have been truly revealed [1]. Epigenetics is defined as the study of changes in gene function, which are mitotically and/or meiotically heritable, but do not cause changes to DNA sequences. For this reason, the process is reversible, which is a further attraction for drug development. Epigenetic modifications comprise DNA methylation, histone modifications and RNA interference (RNAi). DNA methylation affects the promoter region of genes resulting in up- or down-regulation of gene expression. Histone modifications induce or repress transcription. Likewise, RNAi, specifically miRNAs, are postulated to be responsible for the regulation of a third of human mRNAs. Incorrect epigenetic regulation has been associated with metabolic, cardiovascular and neurological diseases as well as cancer [2]. In this commentary, examples of applications of epigenetics for drug discovery are presented.

\section{DNA methylation}

Several approaches have been investigated to develop DNMT inhibitors such as azacytidine and decitabine for cancer therapy [2]. Recently, DNMT inhibitors targeting DNMT3A were discovered by application of structure-based virtual screening in combination with biological assays [3]. Compound 40 and 40_3 showed comparable in vitro inhibition against DNMT3A through binding to $S$-adenosyl-L-methionine and may serve as scaffolds for further optimization of drug molecules. In another study, efficient inhibition was described for the PRMT2 and PRMT4 complex crystal structures, which represents the first step for PRMT2 substrate recognition and may provide an approach for structure-based drug design of PRMT2 inhibitors [4]. In attempts to establish a detection system for DNA demethylating agents suitable for high-throughput screening in mammalian cells, luciferase and EGFP reporter genes were inserted downstream from the UCHL1 promoter, which is methylation silenced in human colon cancers and can be demethylated to drive strong expression [5]. Screening of transfectants from HCT116 colon cancer cells identified three optimal subclones expressing luciferase and EGFP after 5-aza-dC and 5-aza-CR treatments demonstrating the usefulness of this approach to discover epigenetic drugs. In another approach, a technology for large-
Kenneth Lundstrom

'PanTherapeutics, Rue des Remparts 4, CH1095 Lutry, Switzerland Tel.: +41797766351 lundstromkenneth@gmail.com 
scale screening of epigenetic drug candidates with potent hypomethylation activity has been developed for $2 \mathrm{D}$ and $3 \mathrm{D}$ cell culture models [6]. The system utilizes the FLJ32130 fluorescence gene for the evaluation of promoter hypermethylation in human colorectal cancer cells and can also be applied for in vivo monitoring of demethylation efficacy of lead compounds in tumor xenografts. Additionally, it is possible to measure induced cytostatic/cytotoxic drug effects in living cells. In an attempt to investigate the cancer epigenome as a target for innovative therapy, patientderived xenografts (PDXs) of high-grade serious ovarian cancer (HGSOC) were evaluated in relation to the corresponding patient tumor methylome [7]. The outcome was that only $0.6-1.0 \%$ of analyzed CpGs showed a significant change, indicating the epigenetic stability of HGSOC PDXs. Decitabine treatment of PDX tumors resulted in the identification of several putative epigenetically regulated pathways. Particularly, the CSK gene demonstrated epigenetic regulation in different PDX models and ovarian cancer cell lines. Strong associations of both low CSK methylation and high CSK expression with improved progressionfree survival and overall survival in high-grade serious ovarian cancer patients were observed.

\section{"Relatively recently, the epigenetic function of RNAi and especially miRNAs has received signifi- cant attention as part of drug development and diagnostics."}

\section{Histone modifications}

In the context of epigenetic drugs, HDAC inhibitors have been developed in the areas of oncology, cardiology and neurological disorders. Moreover, as differential histone acetylation states occur during the Trypanosoma cruzi cell cycle epigenetic control by HDAC inhibitors for trichostatin A and sirtinol and the activator resveratrol were tested on proliferative and infective parasite forms [8]. The HDAC inhibitors caused life stage-specific effects increasing trypomastigote infectivity, blocking metacyclogenesis and affected specific transcript levels. Resveratrol showed strong antiparasitic effects suggesting that the HDAC inhibitors and resveratrol are potentially promising trypanocidal drug candidates.

Related to epigenetic cancer therapy, HDAC inhibitors such as scriptain, YCW1, santacruzamate A and ferrocenyl have demonstrated promising antitumor activity against breast cancer [9]. Moreover, epigenetic approaches to treat prostate cancer have been restricted to castrate-resistant prostate cancer [10]. Administration of the HDAC inhibitor panobinostat provided stable disease in two clinical trials and in one of them the profile of unfavorable circulating tumor cells was converted to a favorable one in $64 \%$ of the patients. Furthermore, curcumin an HDAC inhibitor from the spice, turmeric, was evaluated in combination with radiotherapy in prostate cancer patients [11]. Although no prostate-specific antigen (PSA) response was obtained, the common side effects of urinary symptoms after radiotherapy were significantly reduced. As several synthetic HDAC inhibitors cause side effects such as atrial fibrillation, the focus has been turned to plant-derived HDAC inhibitors like apigenin [12]. The action of Apigenin occurs through multiple mechanisms including restraining of HDACs and declining of antiapoptotic protein levels. Apigenin has also been confirmed to show synergistic effects after combination therapy and can induce apoptosis in prostate cancer models resistant to conventional therapy.

Recently, DNA demethylating agents and HDAC inhibitors have been used in combination therapy for schizophrenia [13]. In this context, clozapine-induced DNA demethylation was potentiated by the HDAC inhibitor, valproate. In attempts to discover new molecular entities, which enhance epigenetic therapy, high-throughput screening of US FDA approved libraries of DNMT and HDAC inhibitors has been conducted [14]. The YB5 human colon cancer cell line containing the epigenetically silenced cytomegalovirus-green fluorescence protein (CMV-GFP) locus was engineered to mimick tumor suppressor genes, which allowed quantitative monitoring of epigenetic activity by GFP fluorescence. Enhanced DNMT and HDAC inhibitor activities were discovered for 45 FDAapproved drugs ( $4 \%$ of tested) from the FDA-approved libraries mainly in the anticancer and antiarrhytmic drug classes. Furthermore, transcriptome analysis confirmed that the DNMT inhibitor decitabine and the antiarrhytmic drug proscillaridin A caused reprogramming of gene expression including downregulation of 153 epigenetic regulators. Moreover, 85 FDA-approved drugs showed antagonism against DNMT and HDAC inhibitor activities through cytotoxic mechanisms.

\section{RNA interference}

Relatively recently, the epigenetic function of RNAi and especially miRNAs has received significant attention as part of drug development and diagnostics. For instance, circulating miRNAs are easily detectable in body fluids and have demonstrated great potential as noninvasive biomarkers for various diseases including cancer [15]. In this context, miRNA levels have been measured in serum and screening of aberrant miRNA hypermethylation analyzed. Diagnostic tests have been established for miRNAs, which do not only detect drug-induced liver injury, but also 
can differentiate between different drug-induced liver injury types [16]. Application of next generation high-throughput sequencing of small RNA libraries revealed 36 miRNAs including three novel miRNAlike small nuclear RNAs enriched in the serum of accidental acetaminophen overdosed subjects [17]. The discovered miRNAs were functionally associated with liver-specific biological processes.

Furthermore, miRNAs have also caught the attention as therapeutic agents. For instance, miR-135b is commonly upregulated in sporadic and inflammatory bowel disease-associated human colon colorectal cancers [18]. The upregulation correlated with tumor stage and poor clinical outcome and miR-135b was identified as a key downstream effector of oncogenic pathways and therefore presents a potential target for therapy. Neurological disorders are also affected by epigenetics indicated by the high presence of miRNAs in neurons, which has resulted in development of miRNA-based drugs [19]. Related to the recent Ebola epidemic, miRNA precursors and miRNAs have been identified for Ebola virus [20]. This will support the development of noninvasive diagnostic biomarkers and also therapeutic approaches for the treatment of Ebola virus infections.

In the context of clinical trials, antisense oligonucleotides have generated positive PSA responses of $>50 \%$ in three human studies [19]. Furthermore, when combination therapy with docetaxel or mitoxantrone was carried out, a PSA decrease of $>50 \%$ was observed in six of 14 patients with castrate-resistant prostate cancer. In another Phase II clinical trial, the antisense oligonucleotide LY2181308 was applied to decrease expression of the antiapoptotic gene survivin involved in therapy resistance. However, the treated patients showed a higher incidence of grade 3 and 4 adverse events without any improvement in progression-free or overall survival. A Phase I clinical trial was initiated on advanced and metastatic renal cell carcinoma patients with miRNA mimics of the tumor suppressor miR-34. However, the trial was terminated early due to serious immunological adverse events. MRX34, the liposome-formulated mimic of the tumor repressor miR-34 showed complete tumor regression in an orthotopic mouse model and in a Phase I clinical trial on patients with advanced solid tumors, the toxicity profile was manageable. Additionally, evidence of strong anticancer activity was seen in melanoma, hepatocellular and renal cell carcinoma patients. However, the trial was recently put on hold due to multiple immune-related severe adverse events. The locked nucleic acid Miravirsen against miR-122 has been subjected to clinical trials for the treatment of hepatitis C (HCV) [21]. A Phase I study showed good tolerance and dose-dependent pharmacology of the antagomiR-122 and in patients with chronic HCV-1 dose-dependent decrease in HCV RNA levels and no manifestation of viral resistance was observed.

\section{Epigenetic drugs}

There are a number of epigenetic drugs approved [22]. For instance, romidepsin, a cyclic peptide from Chromobacterium violaceum has been approved for the treatment of T-cell lymphoma [23] and also evaluated for pancreatic, colorectal, renal, bladder, brain, thyroid and ovarian cancer. The class III HDAC sirtuin activators and inhibitors have been indicated as potential therapeutic targets in metabolic disease, neurodegeneration and aging [24]. For instance, the sirtuin activator resveratrol found in grapes has been used as a dietary supplement although there is little scientific evidence of its therapeutic efficacy. In this context, a Chinese metaanalysis found a weak association between high-dose resveratrol supplementation and reduced systolic blood pressure [25]. However, no evidence of efficacy was obtained for resveratrol in cancer or metabolic disease therapy. Moreover, curcumin, a p300/CREB-binding protein-specific inhibitor of acetyltransferase, found in curry powder has showed some efficacy in treating Alzheimer's disease, psoriasis, cardiovascular disease and various cancers [26]. Drugs such as decitabine and azacitidine are DNMT inhibitor based and have been approved by the FDA for therapeutic applications of myelodysplastic syndromes [27]. Other DNMT inhibitors include hydrazaline and procainamide, which have been approved for hypertension and cardiac arrhythmia treatment, respectively. Furthermore, several natural products can provide inhibition of both DNMTs and HDACs including the bisulfide bromotyrosine psammaplin from a marine sponge with strong cytotoxicity in human tumor cell lines and tumor growth suppression in a xenograft mouse model [28].

An important part of drug discovery and development relates to bioinformatics and access to available data. Epigenetic drug-related experimental data from high-throughput screening, $\mathrm{x}$-ray structures and bioassays have been stored in the human epigenetic enzyme and modulator database [29]. To further support drug-related issues, the human epigenetic drug database [30] was created, where datasets from laboratory experiments are stored and integrated with the focus on drugs, targets, diseases, high-throughput screening and structural complexes.

\section{Conclusion \& future perspective}

In summary, changes in epigenetic mechanisms including DNA methylation, histone modifications and 
RNAi can result in drastic phenotypic changes leading to development of various diseases. For this reason, development of epigenetic drugs has seen increased interest. The reversible nature of epigenetic modifications provides further basis for serious investments in epigenetic drugs as the changes are not permanent. Moreover, the exposure to environmental factors such as toxic products and inappropriate nutrition (not only malnutrition but also overeating) can trigger the change in epigenetic regulation. Furthermore, DNA methylation and histone modifications have been demonstrated to generate synergistic action resulting in multiple forms of epigenetic regulation and modifications. This so-called 'epigenetic code' could be responsible for the expression of particular sets of genes and also for providing an on/off switch for various cellular events. In this context, epigenetic drugs may prevent the formation of cancer progenitor cells, while killing drug-resistant cancer cells. Although several epigenetic drugs have been approved by the FDA and a number

\section{References}

1 Dupont C, Armant RD, Brenner CA. Epigenetics: definition, mechanisms and clinical perspective. Semin. Reprod. Med. 27(5), 351-357 (2009).

2 Jones PA, Baylin SB. The epigenomics of cancer. Cell 128(4), 683-692 (2007).

3 Shao Z, Xu P, Xu W et al. Discovery of novel methyltransferase $3 \mathrm{~A}$ inhibitors via structure-based virtual screening and biological assays. Bioorg. Med. Chem. Lett. 27(2), 342-346 (2016).

4 Cura V, Marechal N, Troffer-Charlier N et al. Structural studies of protein arginine methyltransferase 2 reveal its interactions with potential substrates and inhibitors. FEBS J. 284(1), 77-96 (2016).

5 Okochi-Takada E, Hattori N, Ito A et al. Establishment of a high-throughput detection system for DNA methylating agents. Epigenetics doi:10.1080/15592294.2016.1267887 (2016) (Epub ahead of print).

6 Agrawal K, Das V, Otmar M, Krečmerová M, Džubák P, Hajdúch M. Cell-based demethylation detection system for screening of epigenetic drugs in 2D, 3D and xenograft models. Cytometry A doi:10.1002/cyto.a.23004 (2016) (Epub ahead of print).

7 Tomar T, de Jong S, Alkema NG et al. Genome-wide methylation profiling of ovarian cancer patient-derived xenografts treated with the demethylating agent decitabine identifies novel epigenetically regulated genes and pathways. Genome Med. 8(1), 107 (2016).

8 Campo VA. Comparative effects of histone deacetylases inhibitors and resveratrol on Trypanosoma cruzi replication, differentiation, infectivity and gene expression. Int. J. Parasitol. Drugs Drug Resist. 7(1), 23-33 (2016).

9 Damaskos C, Valsami S, Kontos M et al. Histone deacetylase inhibitors: an attractive therapeutic strategy against breast cancer. Anticancer Res. 37(1), 35-46 (2017). of clinical trials are in progress, there are still numerous possibilities to develop novel drugs by targeting epigenetic mechanisms. This is particularly relevant to RNAi as our understanding of the involvement of miRNAs in gene regulation and disease increases. Most importantly, recent efforts in bioinformatics and dataset collection in the form of human epigenetic enzyme and modulator database and human epigenetic drug database will provide the basis for future epigenetic drug discovery and development.

\section{Financial \& competing interests disclosure}

The author has no relevant affiliations or financial involvement with any organization or entity with a financial interest in or financial conflict with the subject matter or materials discussed in the manuscript. This includes employment, consultancies, honoraria, stock ownership or options, expert testimony, grants or patents received or pending, or royalties.

No writing assistance was utilized in the production of this manuscript.

10 Faleiro I, Leao R, Binnie A, Andrade de Mello R, Maia A-

T, Castelo-Branco P. Epigenetic therapy in urologic cancers: an update on clinical trials. Oncotarget doi: 10.18632/ oncotarget.14226 (2016) (Epub ahead of print).

11 Hejazi J, Rastmanesh R, Taleban F-A, Molana S-H, Ehtejab G. A pilot clinical trial of radioprotective effects of curcumin supplementation in patients with prostate cancer. J. Cancer Sci. Ther. 5, 320-324 (2013).

12 Ganai SA. Plant-derived flavones apigenin: the small molecule with promising activity against therapeutically resistant prostate cancer. Biomed. Pharmacother. 85, 47-56 (2017).

13 Duenas-Gonzalez A, Coronel J, Cetina L, Gonzalez-Fierro A, Chavez-Blanco A, Taja-Chayeb L. Hydralazine-valproate: a repositioned drug combination for the epigenetic therapy of cancer. Expert Opin. Drug Metab. Toxicol. 10(10), 1433-1444 (2014).

14 Raynal NJ, Da Costa EM, Lee JT et al. Repositioning FDA-approved drugs in combination with epigenetic drugs to reprogram colon cancer epigenome. Mol. Cancer Ther. 16(2), 397-407 (2016).

15 Wang WT, Chen YQ. Circulating microRNAs in cancer: from detection to therapy. J. Hematol. Oncol. 7(1), 86 (2014).

16 Sanjay S, Girish C. Role of miRNA and its potential as a novel diagnostic biomarker in drug-induced liver injury. Eur. J. Clin. Pharmacol. 73(4) 399-407 (2016).

17 Krauskopf J, Caiment F, Claessen SM et al. Application of high-throughput screening to circulating miRNAs reveals novel biomarkers for drug-induced injury. Toxicol. Sci. 143(2), 268-276 (2015).

18 Valeri N, Braconi C, Gasparini P et al. MicroRNA-135b promotes cancer progression by acting as a downstream effector of oncogenic pathways in colon cancer. Cancer Cell 25(4), 469-483 (2014). 
19 Shah MY, Ferrajoli A, Sood AK, Lopez-Berestein G, Calin GA. Micro-RNA therapeutics in cancer - an emerging concept. EBiomedicine 12, 34-42 (2016).

20 Liang H, Zhou Z, Zhang S, Zen K, Chen X, Zhang C. Identification of Ebola virus microRNAs and their putative pathological function. Sci. China Life Sci. 57(10), 973-981 (2014).

21 Janssen HL, Reesink HW, Lawitz EI et al. Treatment of HCV infection by targeting microRNA. N. Engl. J. Med. 368, 1685-1694 (2013).

22 Heerboth S, Lapinska K, Snyder N, Leary M, Rollinson S, Sarkar S. Use of epigenetic drugs in disease: an overview. Genet. Epigenet. 6, 9-19 (2014).

23 Coiffier B, Pro B, Prince HM, Foss F, Sokol L, Greenwood $\mathrm{M}$ et al. Results from a pivotal, open-label, Phase II study of romidepsin in relapsed or refractory peripheral T-cell lymphoma after prior systemic therapy. J. Clin. Oncol. 30(6), 631-636 (2012).

24 Carafa V, Nebbioso A, Altucci L. Sirtuins and disease: the road ahead. Front. Pharmacol. 3, 4 (2012).
25 Poulsen MM, JØrgensen JO, Jessen N, Richelsen B, Pedersen SB. Resveratrol in metabolic health: an overview of the current evidence and perspectives. Ann. NY Acad. Sci. 1290, 74-82 (2013).

26 Balasubramanayam K, Varier RA, Altaf M et al. Curcumin, a novel p300/CREB-binding protein-specific inhibitor of acetyltransferase, represses the acetylation of histone/nonhistone proteins and histone acetyltransferasedependent chromatin transcription. J. Biol. Chem. 279(49), 51163-51171 (2004).

27 Marks PW. Decitabine for acute myeloid leukemia. Expert Rev. Anticancer Ther. 12(3), 299-305 (2012).

28 Baud MG, Leiser T, Haus P et al. Defining the mechanism of action and enzymatic selectivity of psammaplin A against its epigenetic targets. J. Med. Chem. 55(4), 1731-1750 (2012).

29 Shanghai Jiao Tong University. Human Epigenetic Enzyme and Modulator Database. http://mdl.shsmu.edu.cn/HEMD/

30 Human Epigenetic Drug Database. http://hedds.org/ 